\title{
El concepto de alienación en Kierkegaard: análisis del concepto ético de desesperación como descripción kierkegaardeana de la vida dañada
}

\author{
Pablo Uriel Rodríguez \\ Universidad de Morón
}

Resumen: O lo uno o lo otro, la primera obra pseudónima de Kierkegaard, comienza con una exposición de la visión estética de la vida (primer volumen), seguida por la crítica ética de la concepción estética de la existencia (segundo volumen). La categoría de "desesperación" es el concepto central en el rechazo de la estética: la afirmación básica de $\mathrm{O}$ lo uno o lo otro II es que la vida estética es desesperación, y la vida ética ofrece un escape de la desesperación. Este artículo se propone presentar la primera conceptualización kierkegaardeana de la desesperación como una teoría de la alienación.

Palabras clave: Kierkegaard; desesperación; alienación; ética; estética

Abstract: "Kierkegaard's Concept of Alientation: an Analysis of the Ethical Concept of Dispair as the Kierkegaardean Description of Damaged Life". Either/Or, Kierkegaard's first pseudonymous work, begins with an exposition of the aesthetic view of life (first volume), followed by the ethical critic of the aesthetic conception of existence (second volume). The category of despair is the main concept in the rejection of aesthetic: the basic claim of Either/Or II is that the aesthetic life is despair, and the ethical life offers an escape from despair. This paper aims to present Kierkegaard's first conceptualization of despair as a theory of alienation.

Keywords: Kierkegaard; despair; alienation; ethic; aesthetic 
Desde hace doscientos años el concepto de "alienación" viene jugando un papel fundamental en aquellas investigaciones filosóficas que centran su interés en el desarrollo del ser humano y la dinámica de su vida en sociedad. Ya sea explícita o implícitamente, de forma intencional o de manera involuntaria, esta categoría se utiliza en la mayoría de los análisis que exponen y critican la estructura y el funcionamiento de la cultura y la civilización moderna. Incluso cuando la voz danesa "Fremmedgørelse", que traduce los términos alemanes "Entfremdung" y "Entäusserung", no sea empleada por Kierkegaard", los diversos pasajes en los cuales el pensador danés enjuicia a su época y al modo de vida de sus contemporáneos no constituyen una excepción a lo dicho. Para hacer visible y problematizar lo que entiende por una existencia humana patológica, Kierkegaard eleva al rango de conceptos filosóficos una constelación de fenómenos anímicos. A través del análisis del "aburrimiento", la "melancolía", la "pena", la "angustia", etcétera, sus diversos pseudónimos procuran exponer las contradicciones inherentes a la existencia humana desde un punto de vista inmanente, es decir, desde la dolorosa perspectiva de una vida que se experimenta a sí misma dañada ${ }^{2}$. La investigación más articulada, continua y profunda de la situación concreta del ser humano es aquella que se conduce a través del concepto de "desesperación". Esta categoría debuta en las páginas de la segunda parte de O lo uno o lo otro en 1843, reaparece en el volumen inicial de Las obras del amor de 1847 y alcanza su punto máximo de desarrollo y densidad teórica en la primera parte de La enfermedad mortal de 1849. Pese a las transformaciones que sufre a lo largo del tiempo, dentro de la economía argumentativa del danés, el concepto de "desesperación" es la

1 Para las citas de Kierkegaard utilizamos la última edición de sus obras completas indicando en números arábigos tanto el volumen como la página: Søren Kierkegaard Skrifter (SKS), Cappelørn, N.J., Garff, J., Kondrup, J., McKinnon, A., y F.H. Mortensen (eds.), Copenhague, Søren Kierkegaard Forskningscenteret y Gads Forlag, 1997-2009 (55 volúmenes entre textos y comentarios). Ofrecemos, también, la paginación correspondiente de las traducciones al castellano consignadas a continuación: Kierkegaard, S., Escritos de Søren Kierkegaard. O lo uno o lo otro. Un fragmento de vida I, Saez Tajafuerce, B. y D. González (trads.), Madrid: Trotta, 2006 [OO I]; Kierkegaard, S., Escritos de Søren Kierkegaard. O lo uno o lo otro. Un fragmento de vida II, González, D. (trad.), Madrid: Trotta, 2007 [OO II]; Kierkegaard, S., Johannes Climacus o el dudar de todas las cosas, Dip, P. (trad.), Buenos Aires: Gorla, 2007 [JC] y Kierkegaard, S., La enfermedad mortal, Rivero, D. (trad.), Madrid: Sarpe, 1984 [EM].

2 Cf., SKS 2, 216 / OO I, 234. 
categoria kierkegaardeana que funciona en mejor medida como equivalente teórico del concepto hegeliano y post-hegeliano de "alienación".

Numerosos y diversos son los estudios que los especialistas en la obra del danés han dedicado a clarificar el concepto de desesperación. Sin embargo, la gran mayoría de estas investigaciones se enfocan en el tratamiento que esta categoría recibe en el libro de 1849 publicado bajo la pluma del pseudónimo Anti-Climacus. El presente artículo, por el contrario, opta por dirigir su atención al modo en que dicho concepto es tematizado en el segundo volumen de O lo uno o lo otro. Su principal objetivo es reconstruir aquella primera elaboración kierkegaardeana del concepto de desesperación que no contempla los presupuestos fundamentales de la concepción cristiana de la vida: la categoría de pecado y la categoría de "ante Dios". La estructura de nuestro trabajo es simple: (i) comenzamos con unas breves consideraciones etimológicas sobre el término danés "Fortvivlelse"; a continuación (ii) exponemos las objeciones que el autor ético dirige a la concepción estética de la existencia como también su propuesta para la superación de las falencias inherentes a ese modo de vida; y, por último, (iii) ofrecemos una descripción de la desesperación como alienación.

\section{Comprensión preliminar de la desesperación}

El punto de partida de nuestro análisis es una consideración etimológica del término danés "Fortvivlelse" . La palabra está conformada por la raíz "tvivl" que significa "duda" y el prefijo "fort" que sugiere la idea de una mayor intensidad. ¿Reviste esta consideración etimológica algún tipo de importancia en la elaboración que Kierkegaard hace de este concepto? La respuesta a esta cuestión aparece en un valioso pasaje de la segunda carta del pseudónimo ético en el que se define la desesperación como "duda de la personalidad"4. La desesperación es una duda intensificada en el sentido de que ella afecta todas las dimensiones de la existencia del ser humano y no únicamente su faceta o aspecto intelectual. Lejos aún de ser transparente, la expresión "duda de la personalidad" precisa de una mayor aclaración y, en este sentido, es conveniente detenerse en la palabra "duda". En su escrito inconcluso Johannes Climacus o el dudar de todas las cosas, redactado en los primeros meses de 1843, Kierkegaard comienza señalando que en la mayoría de los idiomas existe una relación entre el término

\footnotetext{
3 Nos orientamos, en este punto, a partir de Beabout, G., Freedom and its Misuses: Kierkegaard on Anxiety and Despair, Milwauke: Marquette University Press, 1996, pp. 71-73 y McCarthy, V., The Phenomenology of Moods in Kierkegaard, Boston: Martinus Nijhoff, 1978, pp. 84-87.

4 SKS 3, 203 / OO II, 193
} 
"duda" (tvivle) y el término "dos" (tve), concluyendo a partir de ello que el acto de dudar supone la puesta en relación conflictiva de dos instancias ${ }^{5}$. Atentos a los resultados de este acercamiento de índole etimológico es posible acceder a una caracterización preliminar de la desesperación, que debería confirmarse en nuestra reconstrucción del texto kierkegaardeano: la desesperación consiste en el desdoblamiento o duplicidad (duda) de la personalidad. Notemos que el campo semántico que nuestro idioma reserva para la palabra "desesperación", en principio, parece no tener conexión alguna con esta delimitación provisoria. El hispanoparlante o bien asocia el término "desesperación" con una pérdida total o parcial de expectativas o esperanzas, o bien, en un uso más coloquial, emplea la palabra como sinónimo de impaciencia. Es necesario reconocer que el concepto kierkegaardeano de "desesperación", especialmente en la formulación que recibe en La enfermedad mortal ${ }^{6}$, no es ajeno a los sentidos que le asigna nuestra lengua y ello porque es posible concebir tanto al individuo desesperanzado como al impaciente como víctimas de un sufrimiento existencial producto de una suerte de desdoblamiento temporal de su personalidad: el primero de ellos habita un presente carente de futuro, al segundo el tiempo que separa el presente del porvenir le resulta insoportable. Con todo, no puede dejar de admitirse que al traducir "Fortvivlelse" por "desesperación" perdemos una parte importante de la carga significativa que Kierkegaard tuvo a su disposición a la hora de elaborar filosóficamente su concepto.

\section{Crítica y superación ética del modo de existencia estético}

El más temprano intento por conceptualizar el carácter negativo de la existencia humana lo encuentra el lector de la obra de Kierkegaard en un ensayo titulado El más desdichado. Ubicado en el centro del primer volumen de $O$ lo uno o lo otro, las páginas de este escrito contienen una reflexión teórica del sujeto estético sobre los limites de su propia forma de existencia ${ }^{7}$. Si bien el pseudónimo de turno no utiliza la palabra "desesperación", su presentación

5 Cf. SKS 15, 56-57 / JC, 76. En el escrito aludido Kierkegaard plantea que la existencia de dos instancias es la condición de posibilidad de la duda, mientras que la existencia efectiva del dudar implica un tercero: la relación que pone en contacto las dos instancias mencionadas ( $c f$. SKS 15, 56-57 / JC, 76).

6 Especialmente en la sección "La desesperación de la necesidad equivale a la carencia de posibilidad" del Capítulo 1, Libro III de la Primera Parte (SKS 11, 153-157 / EM, 68-73).

7 Cf., Heller, A., "Fenomenología de la conciencia desdichada. Sobre la función histórica de $L a$ alternativa de Kierkegaard", en: Heller, A., Crítica de la Ilustración. Las antinomias morales de la razón, López Soria, J. (trad.), Barcelona: Península, 1999, p. 153. 
de la conciencia desdichada nos sirve, por una parte, como testimonio inicial a favor de nuestra caracterización provisoria de aquella categoría y, por otra parte, como puerta de entrada a los argumentos esgrimidos por el autor ético en el segundo volumen del libro de 1843. Tras blanquear su deuda con la filosofia hegeliana, el pseudónimo estético nos dice que el individuo desdichado tiene "su ser propio [Egentlige Væsen"] de algún modo fuera de sí [udenfor sig]... está siempre ausente de sí mismo, nunca está presente en sí mismo"9. Al profundizar la caracterización de esta figura existencial, el escrito estético remite el sufrimiento propio de la individualidad desdichada a una suerte de falta de unidad o disgregación de la personalidad, casualmente, de carácter temporal: el desdichado se pierde a sí mismo porque queda completamente referido a su ser pasado o a su ser futuro. Alejándose de sí en el recuerdo o en la expectativa, en ambos casos la conciencia busca su propio reflejo en una idealidad que carece de realidad.

El autor del segundo volumen de O lo uno o lo otro considera que el pseudónimo estético del primer volumen carece de una auténtica concepción de la vida y, por ende, también está desprovisto de un correcto entendimiento tanto de su propia existencia como de sus déficits. Por este motivo, aun cuando el pseudónimo ético reconoce que su par estético detecta los límites de su propia posición existencial evalúa que el análisis crítico de la vida estética desplegado en sus escritos todavía es incompleto y carece de la suficiente radicalidad. A su entender, la única valoración adecuada del modo de existencia estético es posible solo desde una perspectiva superior. Esta visión más acabada de la vida es la suya, lo que quiere decir que una crítica radical y sin concesiones de la vida estética solo puede realizarla quien se adhiera a una cosmovisión ética de la existencia. Dentro del andamiaje teórico de El equilibrio entre lo estético y lo ético en la formación de la personalidad será, precisamente, la categoría de desesperación ${ }^{10}$ la que opere como núcleo central de la crítica al tipo de vida expuesta en la primera mitad de $O$ lo uno o lo otro ${ }^{11}$. Pese a reconocer el carácter inconsistente e insatisfactorio de la exposición de su interlocutor, tanto en su evaluación de la existencia estética como en la elaboración de su

\footnotetext{
8 También es posible traducir esta expresión como "naturaleza esencial".

9 SKS 2, 216 / OO I, 234.

10 Para un análisis del concepto ético de desesperación puede consultarse el artículo Kosch, M., “'Despair' in Kierkegaard's Either/Or", en: Journal of the History of Philosophy, v. XLIV, 1 (2006), pp. 85-97.

11 Cf. Dip, P., "Estudio Preliminar", en: Kierkegaard, S., Johannes Climacus..., p. 17.
} 
concepto de desesperación, el pseudónimo ético recoge un motivo central del auto-enjuiciamiento estético: la experiencia del estar-fuera-de-sí.

La vida de todo individuo, razona el pseudónimo ético evocando un tópico central de la ética aristotélica, posee una orientación global. En el caso del individuo estético esta finalidad se expresa en una sencilla máxima de acción: "disfrutar de la existencia". Si bien "gozar de la vida" se dice de muchas maneras, "aquel que dice querer gozar de la vida pone siempre una condición, la cual puede ser exterior al individuo, o bien estar en el individuo de manera tal que no se encuentra ante él"12. Al diferenciar entre estas dos alternativas existenciales igualmente estéticas el pseudónimo ético está recuperando la distinción fundamental que se construye a lo largo de todo el primer volumen: un esteticismo inmediato (cuya figura paradigmática es Don Juan) y un esteticismo reflexivo (cuya encarnación máxima es Juan, el Seductor). Dejando las diferencias de lado es posible decir que adhiere a una concepción estética de la vida todo aquel que pone el centro de gravedad de su existencia por fuera de sí mismo. No otra cosa, dirá el pseudónimo ético, significa ser un desesperado: "Toda concepción de la vida que tenga su condición fuera de ella misma, sin embargo, es desesperación"13. A partir de estas dos premisas se sigue el siguiente resultado: "toda concepción estética de la vida es desesperación"14. Con esta conclusión el representante de la posición ética se compromete con una afirmación de importancia capital: incluso aquellos individuos estéticos que experimentan satisfacción en su existencia en realidad viven sumergidos, inconscientemente, en la desesperación. Dicho de otro modo: el individuo estético es, en el mejor de los casos, capaz solamente de un vínculo exitoso de hecho con el mundo, pero no de derecho ${ }^{15}$.

\footnotetext{
12 SKS 3, 175 / OO II, 167. Es importante advertir que la crítica del autor ético no se dirige, en primera instancia, al objetivo que el esteta traza como meta existencial (gozar de la vida), sino, más bien, al modo en que se busca este objetivo (poniendo la condición de este gozo fuera del sí mismo). En este sentido, cabe realizar dos observaciones: i) el éxito de las cartas éticas depende de que su interlocutor estético entienda que el fin que él fija para su vida solo puede ser alcanzado si acepta las premisas éticas y ii) la ética defendida en el segundo volumen de $O$ lo uno o lo otro no pretende excluir la estética sino integrarla dentro de sí.

13 SKS 3, 225 / OO II, 213.

${ }^{14}$ SKS 3, 186 / OO II, 177.

15 "Veamos entonces por qué desesperan; es que descubrieron haber fundado su vida en algo efimero; pero ¿es ésa una razón para desesperar? ¿Ha habido algún cambio esencial en aquello en lo que fundaron su vida?... No ha habido nada nuevo que pudiera ser motivo de un cambio. Si desesperan, por tanto, debe ser porque de antemano estaban desesperados. La única diferencia es que no lo sabian, y ésa es una diferencia totalmente fortuita. Se ve entonces que toda concepción estética de la vida es desesperación, y que todo aquel que vive de manera estética está desesperado, lo sepa o no lo sepa" (SKS 3, 186 / OO II, 177).
} 
Consciente de la existencia de una gran variedad de modos estéticos de vida, el interés principal de la teoría ética recae sobre aquellas individualidades estéticas que manifiestan una relación deficitaria con el mundo. Si retornamos al primer volumen de $\mathrm{O}$ lo uno o lo otro encontramos dos escritos que ilustran, sucesivamente, en primer lugar, la actitud que asume la conciencia estética frente a la realidad cuando esta contradice sus pretensiones y, en segundo lugar, la solución que el esteticismo propone para sobreponerse a dicha situación. Nuevamente en las páginas de $E l$ más desdichado leemos que la conciencia desgraciada experimenta la realidad como un territorio extranjero hostil puesto que para ella el mundo es "el tú con el que mantiene un conflicto"16. ¿Cómo reacciona frente a esta adversidad el individuo estético? Modificando el cómo de su relación con el mundo. La rotación de los cultivos analiza el desencuentro entre el yo y el mundo a través del fenómeno del aburrimiento, por lo cual el problema que queda planteado en este escrito es el de cómo vencer el tedio. Por lo común, el individuo que está aburrido cifra su esperanza de entretenimiento en un objeto determinado y cuando este ya no tiene nada nuevo que ofrecerle pasa al siguiente: "Uno está harto de vivir en el campo -escribe el esteta-y viaja a la capital; uno está harto de su país natal y viaja al extranjero... Uno está harto de comer en vajilla de porcelana y lo hace en vajilla de plata; uno está harto de comer en vajilla de oro y quema la mitad de Roma para contemplar el incendio de Troya"17. Este procedimiento revela el anhelo, la mayor de las veces inconfeso, de relacionarse con un objeto inagotable. Sin embargo, para el pseudónimo estético, la auténtica respuesta a este temple anímico negativo no viene, ni puede venir, de la mano de la realidad puesto que esta siempre queda a la zaga del deseo. El primer paso que debe dar el individuo para salvarse del tedio es la auto-limitación: "El método que yo propongo -explica el pseudónimo estético- no radica en transformar el terreno sino, como la verdadera rotación de cultivos, en cambiar el método de explotación y las clases de simiente. Aquí radica ya el principio de limitación, que es el único que salva en este mundo"18. Preciso es, entonces, contener el impetu que lleva al hombre de objeto en objeto. El segundo paso consiste en descubrir que el verdadero placer no se obtiene del objeto en sí mismo sino de aquello que en este se introduce ${ }^{19}$. Se enuncia, de este modo, la necesidad de una superación todavía estética del esteticismo

\footnotetext{
SKS 2, 219 / OO I, 237.

SKS 2, 281 / OO I, $298-299$.

${ }^{8}$ SKS 2, 281 / OO I, 299.

19 Cf. SKS 2, 288 / OO I, 305.
} 
inmediato: si no es posible gozar de la realidad porque esta se muestra refractaria al deseo, entonces es necesario reconfigurarla imaginariamente. Por lo tanto, la propuesta final que el autor de La rotación de los cultivos ofrece para superar el aburrimiento es un claro ejemplo del modo de vida de aquellos individuos que, de acuerdo con el pseudónimo ético, pretenden poner en sí mismos la condición para gozar de la vida.

De acuerdo con la perspectiva ética, la actitud descrita en La rotación de los cultivos sigue siendo desesperada. La reelaboración artística o poetización de la realidad que vendría a garantizar una existencia lograda depende, ciertamente, de la acción del individuo. Sin embargo, para llevar adelante con éxito esta transfiguración del mundo es necesario poseer una capacidad o talento excepcional ${ }^{20}$, es decir, una condición que el individuo no puede darse a sí mismo: "Hay concepciones que nos enseñan que hay que gozar de la vida; pero la condición para ello está en el individuo mismo, si bien de manera tal que no es él quien la pone. Aquí la personalidad está determinada, en general, como talento... Puede ser que uno no se conforme con el talento en su inmediatez y lo eduque de una manera o de otra, pero la condición para la satisfacción en la vida es el talento mismo, y esta es una condición que no está puesta por el individuo" ${ }^{21}$. Al llamar la atención sobre este punto, el pseudónimo ético torna manifiesto que la sabiduria estética logra formular únicamente una salida elitista para el problema de la alienación humana. Desde el punto de vista ético, la verdadera superación de la desesperación debe ser una posibilidad abierta a todo ser humano y dado que todo ser humano sin importar cuáles sean sus características particulares es capaz de desesperar solo desesperando de modo activo y deliberado es posible superar la desesperación. El individuo que elige la desesperación, en primer lugar, reconoce que es un desesperado, es decir, que tiene el centro de gravedad de su existencia fuera de sí mismo y, en segundo lugar, opta por superar su desesperación, es decir, decide recentrar su existencia en sí mismo o, para decirlo con la más célebre expresión del pseudónimo ético, se elige a sí mismo: "si uno ha elegido de verdad la desesperación, ha elegido en verdad aquello que la desesperación elige: el sí mismo..."22.

\footnotetext{
20 "Su esteticismo lo ha arrancado del mundo, que es donde buscaba su placer; lo ha empujado a una esquina desde la cual se ve obligado a confiar en su ingenio y en su imaginación para impedir que todo se derrumbe, y en su capacidad para disfrutar las cosas a través de la reflexión, para disfrutar la idea de las cosas en vez de las cosas mismas" (Hannay, A., Kierkegaard. Una biografia, Bravo Jordan, N. (trad.), México: Universidad Iberoamericana, 2010, pp. 231-232).

${ }^{21}$ SKS 3, 178 / OO II, 170.

${ }^{22}$ SKS 3, 204 / OO II, 194.
} 
La exposición pseudónima de la auto-elección articula este movimiento existencial en tres momentos complementarios, que no hay que pensar como sucesivos sino como simultáneos. Estos momentos o instancias pueden denominarse: aislamiento, concretización y manifestación. A través del primer momento, el individuo advierte un núcleo esencial dentro de su personalidad: el yo se descubre absoluto, es decir, absuelto de todas sus determinaciones, en otras palabras, capaz de tomar distancia de aquellas marcas que constituyen su particularidad. Esta instancia es necesaria puesto que de fallar en el reconocimiento de su libertad, el individuo comprendería las determinaciones de su personalidad como un destino inmodificable. ¿Qué es lo que sucede si el individuo no realiza este reconocimiento? Se considera a sí mismo como un elemento más del mundo natural atrapado en un orden de causalidad mecánico: "El error consiste en que no ha elegido del modo correcto, y no es que no haya sabido ver su error, sino que se ha visto a sí mismo en y según la determinación de la necesidad; a ese sí mismo, esa personalidad en toda la multiplicidad de sus determinaciones, lo ha visto como parte integrante del curso del mundo, lo ha visto frente al poder eterno cuyo fuego lo ha penetrado sin consumirlo"23. Sin embargo, debe existir un contrapeso para esta libertad radical puesto que si el movimiento de auto-elección quedase detenido en este punto el resultado sería una individualidad aun desdichada. Quien se concentra unilateralmente en esta indeterminación originaria de su ser no puede reconocerse a sí mismo en las determinaciones que la realidad le impone ${ }^{24}$. Por este motivo, se vuelve imprescindible un segundo momento por medio del cual la individualidad gana concreción. Esta instancia supone la aceptación de aquellas determinaciones que habian sido puestas en suspenso en la instancia anterior ${ }^{25}$ : "Llega a ser sí mismo, exactamente el mismo que era antes, hasta en el detalle más insignificante, y sin embargo, llega a ser otro, pues la elección todo lo impregna y todo lo transforma" ${ }^{26}$. En su intento por tomar posesión de su propio ser el individuo descubre que al relacionarse consigo mismo se relaciona a su vez con una serie de determinaciones que siéndole propias lo remiten al mundo. Él se comprende como hijo de su tiempo, miembro de un grupo humano particular, inserto en una serie de vínculos interpersonales con otros concretos, etcétera. De todo ello se

\footnotetext{
SKS 3, 221 - 222 / OO II, 209.

Cf. SKS 3, 206 / OO II, 195-196.

Cf. SKS 3, 239 / OO II, 225

${ }^{26}$ SKS 3, 213 / OO II, 202.
} 
sigue que la reconciliación del individuo consigo mismo no puede prescindir de una reconciliación del individuo con la realidad en la cual está inmerso. Precisamente de eso se trata el tercer momento de la auto-elección. El yo debe expresarse en el mundo procurando lograr la unidad entre lo "interior" y lo "exterior": "El sí mismo que es el objetivo no es un sí mismo meramente personal, sino social, civil [o burgués]. Se tiene, pues, a sí mismo como tarea en una actividad ${ }^{27}$ mediante la cual interviene en las circunstancias de la vida como esta personalidad precisa" 28 . El éxito de este tercer momento se funda sobre la convicción de que aquello que la ética prescribe "pasa a la determinación de las costumbres"29, esto es, en la creencia de que el desarrollo histórico de la sociedad ha cristalizado frente al individuo una serie de instituciones culturales y prácticas intersubjetivas -como el matrimonio, la amistad o el trabajo- que, lejos de oprimirlo, favorecen la constitución de su personalidad. Es importante indicar que sin esta tercera y última instancia no quedaría realmente superada la desesperación. Cuando el pseudónimo ético dice que "la elección originaria sigue estando presente en cada elección posterior" 30 , no hay que entender que con ello se proponga una decisión inicial que defina la personalidad de una vez por todas. Interpretar de ese modo la afirmación del ético implicaría recaer en un planteo abstracto que retira al yo del mundo y la historia. Lo que se quiere decir, más bien, es que la elección de sí mismo se realiza a través de una continuidad de elecciones particulares que configuran la actividad vital del individuo.

\section{Desesperación como auto-relación fallida}

Si únicamente quien se elige a sí mismo es capaz de conformarse como individualidad no desesperada, entonces es posible comprender todo lo que está contenido en el concepto ético de desesperación visualizando las consecuencias que se siguen del hecho de no elegirse correctamente a sí mismo. En este sentido, repasar la reconstrucción de las tres instancias en las cuales se descompone teóricamente el movimiento de auto-elección es lo que permite trazar el inventario de las diversas modalidades de ese estar fuera de sí que

\footnotetext{
${ }^{27}$ La relevancia de este componente práctico inclina al pseudónimo ético a priorizar la expresión "elegirse a sí mismo" por sobre la expresión tradicional "conócete a ti mismo" (cf. SKS 3, 246 / OO II, 231-232).

${ }^{28}$ SKS 3, 250 / OO II, 235.

${ }^{29}$ SKS 3, 243 / OO II, 229.

${ }^{30}$ SKS 3, 210 / OO II, 199.
} 
el pseudónimo ético denomina desesperación. Debe decirse, por tanto, que la categoría ética de la desesperación incluiría los tres efectos negativos que se derivan respectivamente del malogramiento de cada uno de los tres momentos de la auto-elección. Atendiendo a un déficit en el momento de aislamiento habría que hablar de una reducción del ser del hombre (sujeto/libertad) al de las cosas (objeto/sustancia); en conexión con una falla en el momento de concretización de un proceso de indeterminación y abstracción del yo y, por último, en el caso de una insuficiencia en el momento de manifestación de una disparidad, ya parcial, ya total, entre la interioridad y la exterioridad. Lo que tienen en común estos tres fenómenos negativos es que cada uno de ellos revela un comportamiento problemático del individuo consigo mismo, un modo de estar el sí mismo fuera de sí mismo. La desesperación ético-existencial de 1843 anticipa, en este sentido, la desesperación psicológico-religiosa de 1849: en ambos casos la experiencia de la desesperación es la de una auto-relación defectiva. Sin embargo, existe una diferencia fundamental entre una y otra concepción. Mientras que para el autor pseudónimo de La enfermedad mortal la auto-relación defectiva tiene su origen en la falta de relación del yo con el $\mathrm{Otro}^{31}$ que lo ha puesto, para el autor del segundo volumen de $O$ lo uno o lo otro, si el individuo habita impropiamente el mundo y se vincula incorrectamente con sus semejantes, es porque, ante todo, fracasa en su relación consigo mismo.

El pseudónimo ético define la auto-elección como un movimiento que el yo realiza "a partir de sí mismo hacia sí mismo a través del mundo"32. Comentando este pasaje Karl Löwith señala que estamos en presencia de un "movimiento hegelianamente definido, de externalización [Entäusserung] y de recuerdo [Erinnerung]"33. El resultado de nuestro recorrido nos reveló que aquello que en las cartas éticas recibe el nombre de desesperación es justamente la ejecución impropia o interrupción de este movimiento. El yo desesperado es aquel que no logra retornar a sí mismo y, de ese modo, estando escindido queda separado de sí. Esta caracterización final de la desesperación como relación defectuosa del individuo consigo mismo a la cual accedimos tras reconstruir la argumentación del pseudónimo ético viene a confirmar el esbozo preliminar del concepto de desesperación con la cual iniciamos nuestro recorrido. El yo desesperado

\footnotetext{
${ }^{31}$ Cf. SKS 11, 129-130 / EM, 36-37.

${ }^{32}$ SKS 3, 261 / OO II, 245.

${ }^{33}$ Löwith, K., De Hegel a Nietzsche. La quiebra revolucionaria del pensamiento en el siglo XIX. Marx y Kierkegaard, Estiú, E. (trad.), Buenos Aires: Sudamericana, 1986, p. 394. Modificamos ligeramente la versión española de Emilio Estiú que traduce "Entäusserung" por "alienación".
} 
es un yo dubitativo, en conflicto, un yo dividido que no logra reconocerse a sí mismo. Si fuese necesario condensar en una única expresión la experiencia del individuo desesperado podríamos referirnos a él como un "yo disociado". Asumiendo con plena consciencia el riesgo hermenéutico que toda traducción comporta, sería correcto tal vez utilizar la palabra "disociación" para reflejar en nuestro idioma lo que Kierkegaard y sus pseudónimos entendian cuando hablaban de "Fortvivlelse".

\section{Consideraciones finales}

La filósofa húngara Agnes Heller en su artículo de 1976 titulado Fenomenología de la conciencia desdichada afirma que los dos volúmenes de O lo uno o lo otro contienen uno de los ataques más agudos y despiadados a la sociedad europea de la primera mitad del siglo XIX. Sin embargo, la discípula de Georg Lukács añade que la crítica de Kierkegaard permanece dentro de los márgenes de una perspectiva burguesa ${ }^{34}$. En este sentido, consideramos que nuestra reconstrucción del concepto de alienación que elabora el pseudónimo ético a través de la categoría de desesperación confirma este señalamiento de Heller.

La alienación del pseudónimo ético estaria más cerca del concepto hegeliano que del marxiano puesto que lo que distancia al yo de sí mismo, esto es, aquello que lo aliena, es su incapacidad para reencontrarse a sí mismo en lo otro de sí, como en el caso de Hegel, y no el producto de una actividad objetivada del yo que se vuelve contra este por el hecho de no pertenecerle, como en el caso de Marx $^{35}$. En el discurso del Kierkegaard-ético la alienación queda tematizada como un fenómeno subjetivo de carácter espiritual, en Marx la alienación es un fenómeno objetivo de carácter material. Desde una perspectiva marxista, el análisis del danés permanece en un plano individual-existencial sin cuestionar los condicionamientos históricos y económicos que dan origen a la vida alienada. Por este motivo, también difieren las respuestas que ambos pensadores encuentran para esta problemática. Para Kierkegaard no es necesario transse relaciona consigo mismo y a partir de ello renovar la relación del yo con el

\footnotetext{
${ }^{34}$ Cf. Heller A., "Fenomenología de la conciencia desdichada", en: Heller, A., Crítica de la Ilustración. Las antinomias morales de la razón, López Soria, J. (trad.), Barcelona: Península, p.177.

${ }^{35}$ Cf. Wittmann D., "Las fuentes del concepto de alienación" en Renault, E. (comp.), Leer los Manuscritos de 1844 de Marx, Cardoso, H. (trad.), Buenos Aires: Nueva Visión, 2009, p 108.
} 
mundo; para Marx, precisamente, se trata de transformar al mundo, esto es de modificar radicalmente la estructura económica de la sociedad burguesa, para que sea posible una nueva forma de vida humana.

Recibido: $16 / 08 / 2016$

Aceptado: 21/04/2016

\section{Bibliografia}

Beabout, G., Freedom and its Misuses: Kierkegaard on Anxiety and Despair, Milwaukee: Marquette University Press, 1996.

Dip, P., "Estudio Preliminar", en: Kierkegaard S., Johannes Climacus o el dudar de todas las cosas, Dip, P. (trad.), Buenos Aires: Gorla, 2007, pp. 7-38.

Hannay, A., Kierkegaard. Una biografia, Bravo Jordan, N. (trad.), México: Universidad Iberoamericana, 2010.

Heller, A., "Fenomenología de la conciencia desdichada. Sobre la función histórica de La alternativa de Kierkegaard", en: Heller, A., Crítica de la Ilustración. Las antinomias morales de la razón, López Soria, J. (trad.), Barcelona: Península, 1999, pp. 135-177.

Kierkegaard, S., La enfermedad mortal, Rivero, D. (trad.), Madrid: Sarpe, 1984 [EM].

Kierkegaard, S., Escritos de Søren Kierkegaard. O lo uno o lo otro. Un fragmento de vida I, Saez Tajafuerce, B. y D. González (trads.), Madrid: Trotta, 2006 [OO I].

Kierkegaard, S., Escritos de Søren Kierkegaard. O lo uno o lo otro. Un fragmento de vida II, González, D. (trad.), Madrid, Trotta: 2007 [OO II].

Kierkegaard, S., Johannes Climacus o el dudar de todas las cosas, Dip, P. (trad.), Buenos Aires: Gorla, 2007 [JC].

Kosch, M., "'Despair' in Kierkegaard's Either/Or”, en: Journal of the History of Philosophy, v. XLIV, 1 (2006), pp. 85-97. https://doi.org/10.1353/hph.2006.0013

Löwith, K., De Hegel a Nietzsche. La quiebra revolucionaria del pensamiento en el siglo XIX. Marx y Kierkegaard, Estiú, E. (trad.), Buenos Aires: Sudamericana, 1986.

McCarthy, V., The Phenomenology of Moods in Kierkegaard, Boston: Martinus Nijhoff, 1978. https://doi.org/10.1007/978-94-009-9670-0

Wittmann, D., "Las fuentes del concepto de alienación", en: Renault, E. (comp.), Leer los Manuscritos de 1844 de Marx, Cardoso, H. (trad.), Buenos Aires: Nueva Visión, 2009, pp. 103-124. 